\title{
VISUALIZING ALL THE FITS: EVALUATING THE QUALITY AND PRECISION OF PARAMETRIC IMAGES CREATED FROM DIRECT RECONSTRUCTION OF PET SINOGRAM DATA
}

\author{
Evan D. Morris ${ }^{1}$, Mustafa E Kamasak ${ }^{2}$, Bradley T. Christian ${ }^{3}$, Tee Ean Cheng ${ }^{1}$, Charles A. Bouman ${ }^{4}$ \\ 1 Indiana University-Purdue University, Indianapolis, 2. Istanbul Technical University, 3. University of \\ Wisconsin-Madison, 4. Purdue University
}

\begin{abstract}
We have recently implemented and tested the direct reconstruction of sinogram data to dense images of kinetic model parameters [1]. In addition, we have recently applied our algorithms to brain data acquired with $18 \mathrm{~F}$-fallypride imaging of a monkey [2]. As a multi-dimensional parameter estimation exercise, direct reconstruction to parametric images can be thought of as generating thousands of modelfitted curves (the prediction of measured sinograms) simultaneously. Because the resulting parametric images are only as good as the fits to the data, one would like to have a means of evaluating the "goodness of fit" of each of the model-fitted curves. The size of the data set involved (4D PET data) presents unique problems in the visualization of the fits. In this paper, we propose measures to objectively evaluate the "goodness of fit" of the model to the PET sinograms in orders to evaluate the precision of the parametric images and the validity kinetic model. The techniques presented are, in part, extrapolations of standard parameter estimation techniques [3] to multi-dimensional estimates and are adapted to the tomography paradigm.
\end{abstract}

\section{INTRODUCTION}

Direct reconstruction of parametric images is a newly implemented technique for reconstructing PET sinograms that bypasses the creation of emission images and proceeds directly to a more useful end-product, images of physiologically relevant parameters [1]. The conventional ("indirect") approach to the analysis of dynamic PET data involves first a reconstruction step to emission images at multiple time-points, second, region drawing and curve creation, and third, estimation of kinetic parameters from only a sparse (compared to the total number of voxels) selection of spatial locations in the images. The direct approach deals with the data in sinogram space and relies on the incorporation of a kinetic model into the iterative reconstruction framework. The direct approach eliminates the often labor intensive tasks of managing, processing and storing emission images. On the other hand, the direct approach presents new challenges for visualizing the fits of the model to the data. The quality of the fits of the model- predicted sinograms vs the measured sinograms is an essential part of determining whether or not the model that is incorporated into the reconstruction algorithm is an appropriate one.

\subsection{Kinetic and Scanner Models Combined}

The kinetic model that we have included in our direct reconstruction framework is the standard four-parameter, two-tissue compartment model used commonly to describe the uptake and retention of high specific activity receptorligand tracers in PET. The model, shown in Figure 1, requires a measured plasma input function and can be solved via numerical convolution of the input function with the impulse response function. In turn, the kinetic model solved at every voxel, yields voxel-wise estimates of the PET emissions for a given set of kinetic parameter values, i.e., parametric images. The predicted emissions, $f$, at voxel $\mathrm{s}$, over time-frame $\left[\mathrm{t}_{\mathrm{i}}, \mathrm{t}_{\mathrm{j}}\right]$ is a function of kinetic parameters $\varphi_{\mathrm{s}}$ that are unique to voxel s, and are given generically as:

$f\left(\varphi_{s}\right)_{t_{i}}^{t_{j}}=\frac{1}{\Delta t} \int_{i-j} \int_{t_{i}}^{t_{j}} V_{B} C_{W B}(s, t)+\left(1-V_{B}\right) S A(t) \sum_{l=1}^{n}\left[C_{l}(s, t)\right] d t$

where $\mathrm{SA}(\mathrm{t})$ is the time-varying specific activity of the tracer, $\mathrm{C}_{\mathrm{WB}}$ is activity concentration in the blood, $\mathrm{V}_{\mathrm{B}}$ is the blood volume fraction of the tissue, and $\mathrm{C}_{1}$ is the $l^{\text {th }}$ compartment of the kinetic model (see Figure 1).

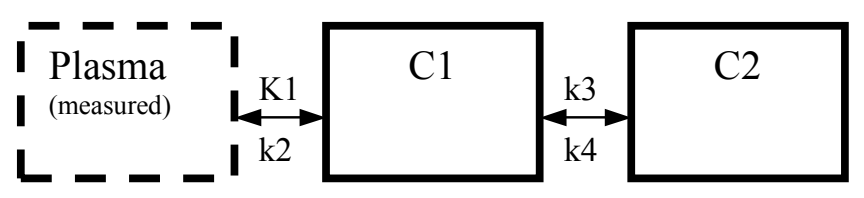

Figure 1. Schematic diagram of two-compartment, 4-parameter model. Plasma radioactivity (dotted box) is measured and therefore not a compartment in the mathematical sense. This model was incorporated into direct reconstruction by the authors (Kamasak et al., 2005).

Within the iterative reconstruction algorithm, the emissions map is projected to sinograms, $\mathbf{Y}_{\mathbf{p}}$, via the scanner model 


$$
\mathbf{Y}_{\mathbf{p}}=\mathbf{A F}(\varphi)
$$

where $\mathbf{Y}_{\mathbf{p}}$ are the predicted sinograms, $\mathbf{F}$ are the emission images and $\mathbf{A}$ is the projection matrix (aka., the scanner model). The parametric images are reconstructed by minimizing the cost function given as

$\boldsymbol{\varphi}=\operatorname{argmin}_{\varphi}[-L L(\boldsymbol{Y} \mid \varphi)+S(\varphi)]$.

where LL is the log likelihood, $\mathbf{Y}$ are the measured sinograms, $\varphi$ is the estimated parametric image, and $S$ represents the regularization kernels that are applied locally to each of the parametric images on each iteration of the minimization procedure. At the end of each iteration, the parametric images are updated until convergence is achieved. Details of the algorithm and its performance on realistic dynamic phantom data are given in [1]. Results from ${ }^{18} \mathrm{~F}$-fallypride neuroimaging of monkeys are given in an accompanying paper [2].

In this paper, we focus on evaluating the results of the reconstruction. That is, how precise are the parametric images at each voxel and how do we know that the kinetic model selected was appropriate to the task?

To answer these questions, we modify some standard practices from 'scalar' parameter estimation. First, as a demonstration of principles, we present variance images for each parameter based on Monte Carlo simulations. The images of the variance -or a related error index- provide an estimate of the precision of the voxel-wise parameter estimates. Without such estimates, it is impossible to say definitively that one image is different from another if the images cannot be acquired multiple times. Second, we introduce and evaluate back-projected images of residuals in sinogram space as a means of visualizing the fits of the model to the data over many times and spatial locations, simultaneously. With the images of the back-projected residuals, an adequate kinetic model can be distinguished from an inadequate model, that is, the residual images are a tool for proper model selection.

\section{METHOD}

\subsection{Image Reconstruction}

Parametric images were reconstructed according to the algorithm described in detail in Kamasak et al., [1,2]. The final images (shown in Figure 2 for parameters $\mathrm{K}_{1}, \mathrm{k}_{2}, \mathrm{k}_{3}, \mathrm{k}_{4}$, were then treated as the 'truth' for the purpose of estimating images of the error.
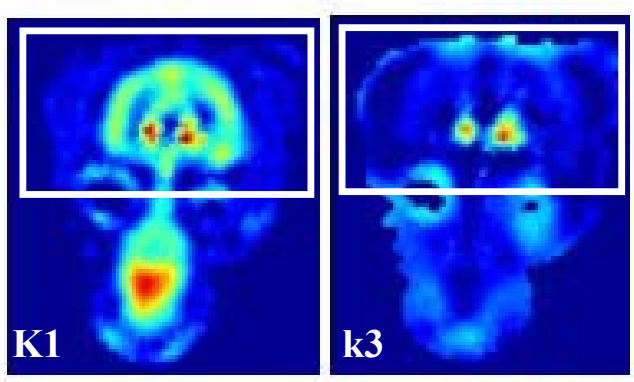

Figure 2.

Parametric

images for $K_{l}$,

$k_{2}, k_{3}, k_{4}$ in

coronal

perspective

created by

minimizing

Equation 3 with

$18 F$-fallypride
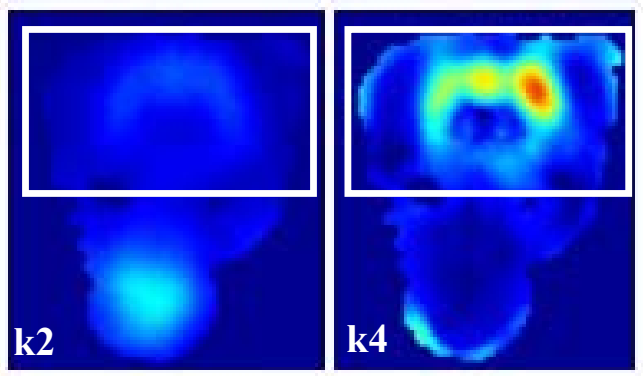

data acquired in

a monkey on an

$H R+$ scanner.

White rectangles

around brain

indicate the area

shown in

Coefficient of

Variation

images (Fig. 5).

\subsection{Creation of Variance Images}

Images of $\mathrm{K}_{1}, \mathrm{k}_{2}, \mathrm{k}_{3}, \mathrm{k}_{4}$ (shown in Figure 2) were input into the forward projection model of our scanner (see Equation 2) and combined with a Poisson noise model that was consistent with the activity in our experimental data. Ten independent realizations of noisy sinogram data were produced and then treated as real data. That is, the simulations were reconstructed to parametric images according to Equation 3. The variance of each parametric image was then calculated voxel-by-voxel from the 10 sets of reconstructed images according to:

$\operatorname{Var}\left(\theta_{i s}\right)=\frac{\sum_{j=1}^{n}\left(\overline{\theta_{i s}}-\theta_{i s}\right)_{j}^{2}}{n-1}$

where $\theta_{i s}$ is the estimate of the $i^{\text {th }}$ parameter at voxel, $\mathrm{s} ; \mathrm{n}$ is the number of independent realizations. We present the variance images below, as coefficient of variation images $\left[\mathrm{CV}\left(\mathrm{K}_{1}\right) \quad \mathrm{CV}\left(\mathrm{k}_{2}\right) \quad \mathrm{CV}\left(\mathrm{k}_{3}\right) \quad \mathrm{CV}\left(\mathrm{k}_{4}\right)\right]$. The coefficient of variation is standard deviation of the value normalized by the value, times 100 . Displaying coefficient of variation images, which are all scaled to $100 \%$, ameliorates scaling problems in the display and visually conveys the percent error in each respective parameter.

\subsection{Back-projection of residuals}

In sinogram space, we can visualize the agreement between the model prediction and the acquired data for every timeframe and angle as a single fitted curve of events vs. distance. An example fit in sinogram space is presented in Figure 3. In order to visualize all the fits in sinogram space (for a single time-frame) simultaneously, we back-project 
the residuals between the fitted (predicted) and measured projections for all angles using filtered-backprojection. Let $\mathrm{R}(\varphi)$ denote the residual sinograms, which are the difference between the fitted and measured sinograms,

$\mathrm{R}(\varphi)=\mathrm{Y}_{\mathrm{p}}(\varphi)-\mathrm{Y}(\varphi)$

and let $\mathrm{e}(\varphi)$ be the filtered backprojection of the residual sinograms.

An example fit to the recorded counts in the sinogram domain (at a fixed projection angle and fixed time) vs. radial distances is shown in Figure 3. The selected projection angle is indicated in Figure $3 \mathrm{a}$ by a white horizontal line across the sinogram. The measured and fitted curves at this particular time and projection angle is shown in Figure 3b. The residuals are given in Figure $3 \mathrm{c}$ as the normalized error between the measured and fitted curves. The error shown in Figure $3 \mathrm{c}$ appears uncorrelated in radial distance which suggests that the kinetic model (in this case a twocompartment model) was adequate to fit the data. Had the residuals been non-random, we would have taken that to indicate a deficiency of the kinetic model [3].
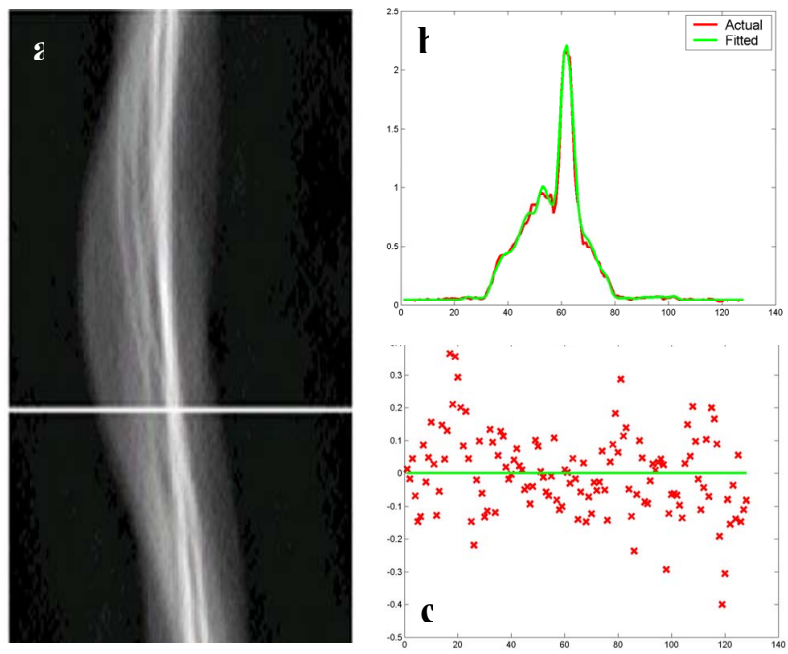

Figure 3 a. Sinogram data; time-frame 30, angle 80 is identified by horizontal line through all distances. $b$. measured sinogram projection vs distance (red) are overlaid on predicted sinogram projection (green). c. Normalized residuals of fit in $b$.

In order to evaluate the utility of the back-projected residuals as a means of model selection, we reconstructed the data (used for Figure 1) in two ways. First, we performed a direct reconstruction that incorporated the 4parameter, two-compartment model alluded to earlier, and second, we preformed direct reconstruction with a 2parameter, one-compartment kinetic model. The onecompartment, or blood flow, model is diagrammed in Figure 4. This model is clearly deficient for describing the uptake of a tracer that binds to a receptor site (requiring a binding compartment), therefore we expect some indication of model deficiency in the back-projected residual images, $\mathrm{e}(\varphi)$.

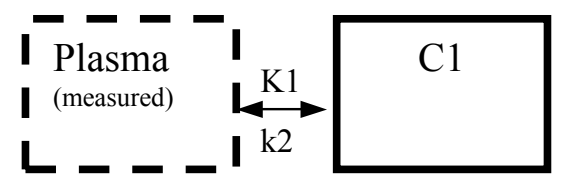

Figure 4. Schematic diagram of one-compartment, 2-parameter model. Plasma radioactivity (dotted box) is a measured function. This model was incorporated into direct reconstruction in order to test the use of back-projected residuals for kinetic mode selection.

\section{RESULTS}

The coefficient of variation images based on our Monte Carlo simulations are shown in Figure 5. Close examination of the images reveals low coefficient of variation in the striatal and cortical regions of the brain. Some bright areas (high percentage variation) in the $\mathrm{k}_{3}$ image correspond to the ventricles consisting only of cerebrospinal fluid and not brain tissue. Surrounding skull and muscle are crosshatched for ease of viewing the brain. The coefficient of variation in $\mathrm{k}_{2}$ parameter is not zero but it is very small. In practical usage, the images could be masked to retain only gray and white matter areas.
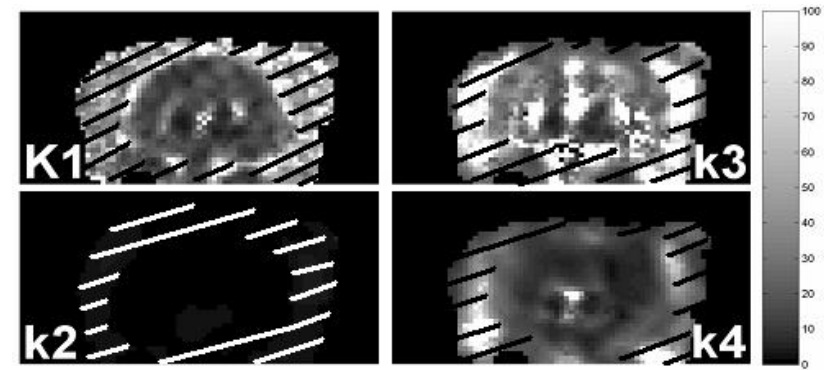

Figure 5. Coefficient of variation $(C V=\operatorname{StdDev}(\theta) / \theta * 100 \%)$ images of $K_{1}, k_{2}, k_{3}$, and $k_{4}$. Variances were estimated based on direct reconstruction of 10 independent noisy realizations of sinogram data projected from the fit in Fig 2. Regularization as described in [2]. Note: Fractional error in parameters within gray matter of brain is small. $C V$ is very high where parameters are 0 or do not exist. $C V\left(k_{2}\right)$ is non-zero but very small.

The error images, $\mathrm{e}(\varphi)$, reconstructed via filtered backprojection from the residual sinograms are shown in Figure 6. The left hand column of Figure 6 shows the emission image at various time frames. The middle and right-hand column show the $\mathrm{e}(\varphi)$ images for the models shown in Figures 1 (two-compartment) and 4 (onecompartment), respectively. On each row, the e $(\varphi)$ image 
corresponds to the time-frame indicated at the left. All error images are displayed using the same color scale. The images in the middle column are generally noisy images without structure. On the other hand, the images in the right hand column show a fair degree of structure that is reminiscent of the corresponding emission images shown at the left. This structure arises from non-random residuals in sinogram space and confirms that the one-compartment model is inadequate to describe the uptake and retention of ${ }^{18} \mathrm{~F}$ fallypride tracer in the brain.

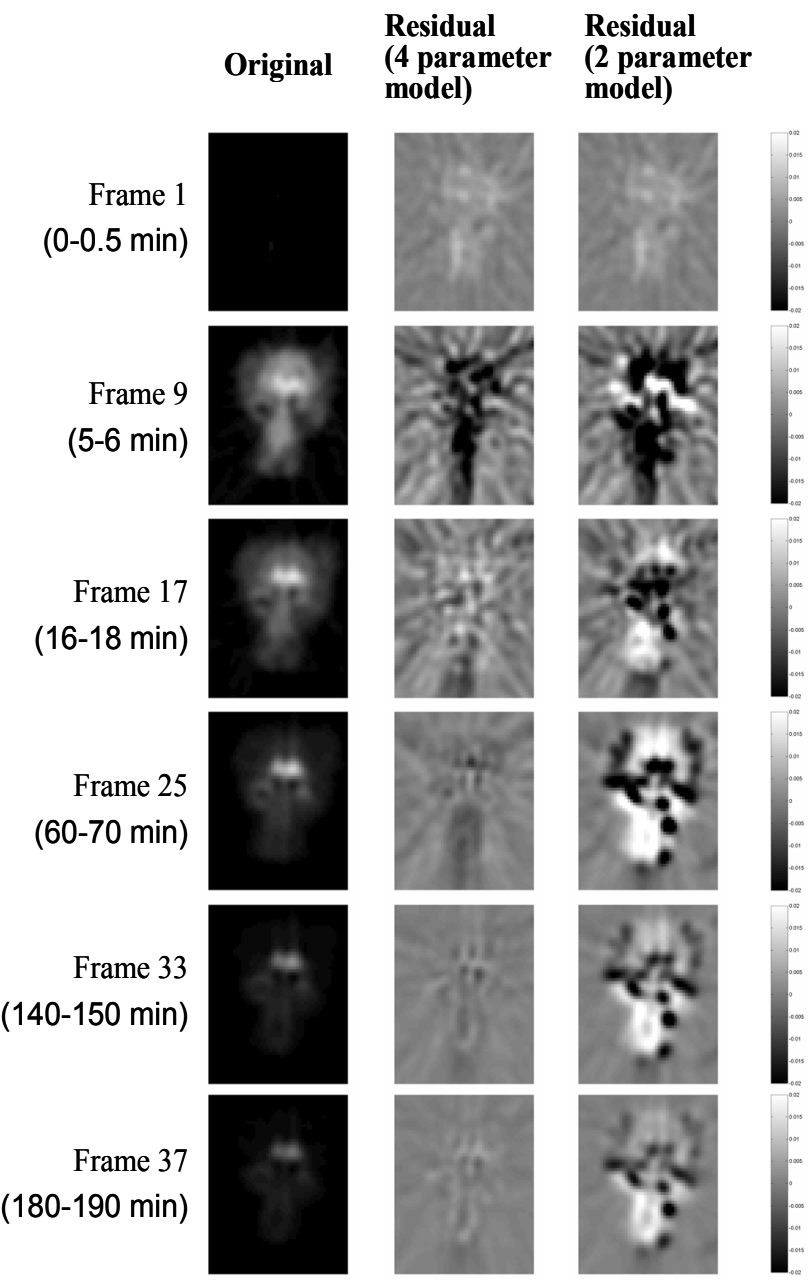

Figure 6. Emission images and back-projected residuals. Left Column: emission images of ${ }^{18} \mathrm{~F}$-fallypride uptake in monkey head; time frames indicated at left. Middle Column: Backprojections of residuals in sinogram space from corresponding time frame. Kinetic model used: 4-parameter, twocompartment, with moderate regularization in all parameters (see [2]). Right Column: Back-projections of residuals in sinogram space from corresponding time frame. Kinetic model used: 2-parameter, one-compartment; moderate regularization in all parameters. All back-projected residuals displayed on same color scale. Note lack of form in Middle column. Apparent object in Right Column suggests non-random residuals in sinogram space and inadequacy of kinetic model.

\section{DISCUSSION and CONCLUSIONS}

The residuals, $\mathrm{R}$, that were used for back-projection to error images, were not normalized. Therefore, the residual sinograms are in units of counts and the backprojected error images (if properly calibrated) are in units of radioactivity. Thus, one can think of the $\mathrm{e}(\varphi)$ images as the remaining activity distribution that cannot be explained by the chosen kinetic model. If such a distribution is highly non-random, then the kinetic model that has been incorporated into direct reconstruction must be deemed inadequate.

In this paper we have introduced two means of verifying the precision and validity of the parametric images created by a recently introduced algorithm for direct reconstruction from sinograms [1]. The variance images are an essential element for assessing the accuracy of any parametric image. As in any experiment, an 'acceptable' variance can only be determined in the context of the expected effect size, number of subjects in a study, etc. Monte Carlo simulations are probably too cumbersome for use as a routine means of calculating the variance images, but they will serve as a check of other methods for estimating variance from the Fisher Information matrix, which we intend to implement.

But even precision is only one aspect of a good result. We also want to know that the kinetic model was capable of describing the data. This cannot be discerned by merely looking at the parametric images and there are far too many projections and time frames to allow for visual inspection of the fits in the sinogram domain. Thus, we sought a means of visualizing all the residuals simultaneously and the natural way to view projection data is to back-project them. The clear difference in the appearance between the error images from the one- and two-compartmental models suggest that such images can be used to help select the appropriate kinetic model for use in direct reconstruction.

\section{REFERENCES}

[1] Mustafa E. Kamasak, Charles A. Bouman, Evan D. Morris, and Ken Sauer, "Direct reconstruction of kinetic parameter images from dynamic PET data," IEEE Trans. on Medical Imaging, vol. 24, no. 5, pp. 636-650, May 2005.

[2] Mustafa E. Kamasak, Charles A. Bouman, Evan D. Morris, and Bradley T. Christian, "Direct reconstruction of parametric images using $18 \mathrm{~F}$-fallypride in non-human primates," submitted to Int. Symp. on Biomedical Imaging, 2006.

[3] Cobelli C, Foster D, Toffolo G, "Tracer Kinetics in Biomedical Research” Kluwer Academic, New York, 2000. 